\title{
Tri-frequency Microcosmic Fractal Dipole Antenna with PBG Structure Used for 2G, 3G and 4G Systems
}

\author{
Bin $\operatorname{LIN}^{1, *}$ \\ ${ }^{1}$ Xiamen University Tan Kah Kee College, Fujian Zhangzhou 363105, China \\ *Email: linbin@xujc.com
}

Keywords: Hilbert fractal structure, Microcosmic fractal structure, Dipole antenna, Mirroring compensation structure, PBG structure, Cantor fractal structure.

\begin{abstract}
Basing on the requirement of the second generation, the third generation and the fourth generation mobile communication systems, a tri-frequency microcosmic fractal dipole antenna is designed with a novel PBG structure. A sample of this antenna has been tested after simulation analysis and discussed the influence of the performance of this antenna by changing the PBG parameter in detail. The simulated and tested results show that this antenna has low return loss, wide working bandwidth and hemisphere direction radiation characteristic, at three working center frequencies of $0.92 \mathrm{GHz}, 1.80 \mathrm{GHz}$ and $2.58 \mathrm{GHz}$. Utilizing fractal structure in PBG, the antenna performance can be improved and the working bandwidth can be broadened effectively. This antenna can completely covered the GSM, TD-SCDMA, WCDMA and TD-LTE communication frequency band; it successfully achieved the compatibility of the second generation, the third generation and the fourth generation mobile communication systems.
\end{abstract}

\section{Introduction}

With the development of mobile communication technology, the second generation $(2 \mathrm{G})$, the third generation $(3 \mathrm{G})$ and the fourth generation $(4 \mathrm{G})$ mobile communication systems will coexist for a long time [1-2]. This requires that the mobile communications antenna should have the multi-band compatibility function, cover the working bands of GSM, TD-SCDMA, WCDMA, TD-LTE and other mobile communications standard. At present, the GSM standard communication bands are $0.905-0.915 \mathrm{GHz}, 0.950-0.960 \mathrm{GHz}, 1.710-1.785 \mathrm{GHz}$ and $1.805-1.880 \mathrm{GHz}$ [3], the TD-SCDMA standard communication bands are $1.880-1.920 \mathrm{GHz}$ and $2.010-2.025$ $\mathrm{GHz}$, the WCDMA standard communication bands are $1.920-1.980 \mathrm{GHz}$ and 2.110-2.170 GHz [4], the TD-LTE standard communication bands are 2.300-2.390 $\mathrm{GHz}$ and $2.555-2.655 \mathrm{GHz}$ [5].

Dipole antenna has been applied widely in mobile communication systems, due to wide working band, low cost and whole direction radiation characteristic [6-8]. However, there are still some deficiencies to be overcome such as weak radiation ability and working only in single-frequency band [9-11].

For the dipole antenna, microcosmic fractal structure is an effective improved structure. The dipole antenna arm has a certain width, the small fractal structure is used in each small square area of the antenna arm, and this microcosmic fractal structure can improve the bandwidth performance of the antenna. Mirror compensation structure has the same structure and size as metal dipole antenna radiation patch, and it is installed symmetrically to metal dipole antenna. By reasonably adjusting the spacing, can make the radiation signal of metal dipole antenna and mirror compensation structure in-phase 
stacking, it can greatly improve the antenna's return loss performance and radiation performance, extend the antenna's working bandwidth. Exploiting some photonic band gap (PBG) structures on the grounded plane of antenna, the radiation signal generated by the antenna arms can let metal PBG structures produce induction radiation. The radiation characteristics of antenna could be strengthened by the induction radiation of PBG [12-13].

Combining microcosmic fractal structure, mirror compensation structure, PBG structure with dipole antenna, we designed a tri-frequency microcosmic fractal dipole antenna with minimized size and better properties for $2 \mathrm{G}, 3 \mathrm{G}$ and $4 \mathrm{G}$ systems, taking ceramic material with high dielectric constant as the substrate.

\section{Brief Introduction of Hilbert Fractal Structure}

The iteration process of Hilbert fractal structure is shown as Figure 1. The initial unit is a vertical three-segment line which composed with three segments with equal length. To get first-order Hilbert Fractal structure, divide each segment into three parts with equal length and construct a small three-segment line which has $1 / 3$ length of initial unit. Clockwise substitute the small three-segment line with the former part of the first segment, the middle part of the second segment and the rear part of the third segment. To get second-order Hilbert Fractal Structure, similarly iterate each three-segment area of the first-order Hilbert Fractal Structure. Iterating as this way, we can get high-order Hilbert fractal structure. Hilbert fractal structure with certain line width has strong space filling ability, and it suitable for the fractal transformation of the small square metal region on the antenna [14-16].

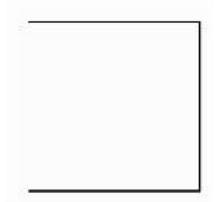

(a) 0-order

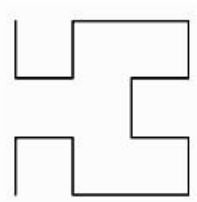

(b) 1 st-order

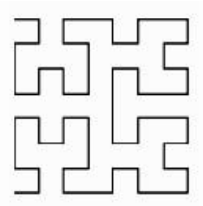

(c) 2nd-order

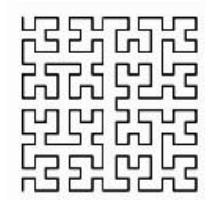

(d) 3rd-order

Figure 1. The iteration process of Hilbert fractal structure

\section{The Structural Design of the Tri-frequency Microcosmic Fractal Dipole Antenna with PBG Structure}

Setting the relative dielectric constant and thickness of ceramic substrate as $\varepsilon_{r}=20$ and $h=4 \mathrm{~mm}$ respectively, we have designed a tri-frequency microcosmic fractal dipole antenna with PBG structure, the size of antenna is $30 \mathrm{~mm} \times 30 \mathrm{~mm}$. The schematic diagram of the antenna structure is shown in Figure 2.

We use the radiation of dipole antenna structure to cover the $0.905-0.915 \mathrm{GHz}$ and 0.950-0.960 GHz frequency bands. The length of dipole antenna arm meets the formula (1).

$$
l_{0}=\frac{c}{4 \sqrt{\varepsilon_{r}} f_{0}}
$$

In these formula, light velocity $c=3.0 \times 10^{8} \mathrm{~m} / \mathrm{s}$, ceramic substrate relative dielectric constant $\varepsilon_{r}=20$. After calculating, when $f_{0}=0.90 \mathrm{GHz}$, the $l_{0}=18 \mathrm{~mm}$. In order to reduce the antenna's size, the antenna arm has been folded, the horizontal arm's length is $9 \mathrm{~mm}$, and the longitudinal arm's length is $9 \mathrm{~mm}$. We use the mirror compensation structure to improve the antenna's radiation performance. 


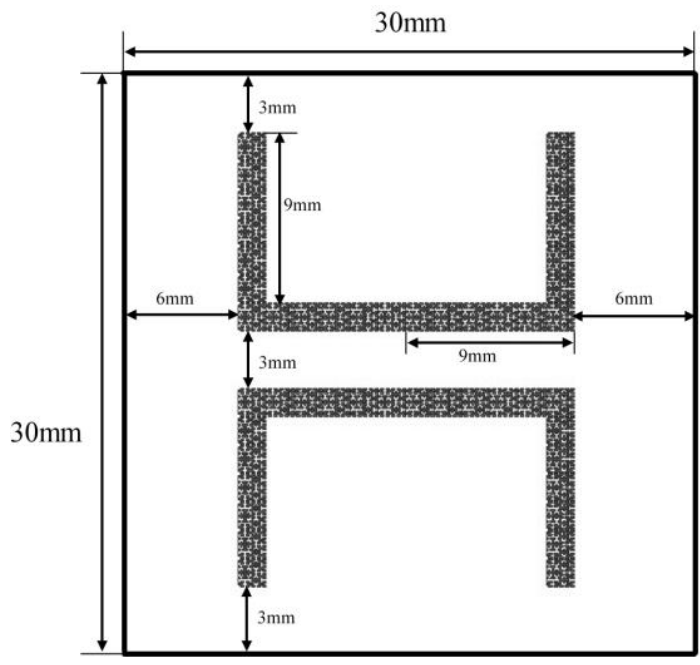

(a) microcosmic fractal dipole antenna structure

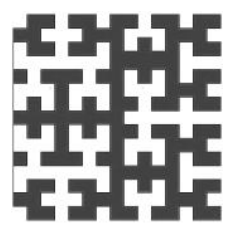

(b) 3rd-order Hilbert fractal structure

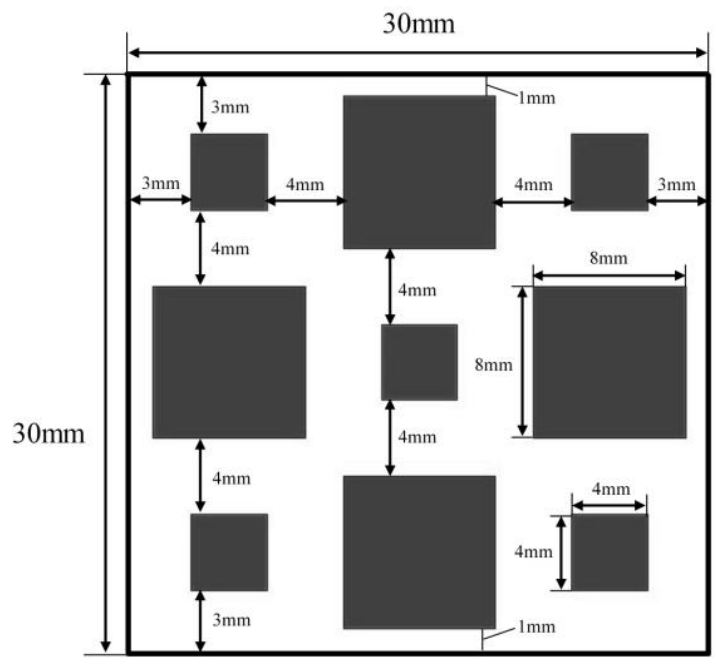

(c) PBG structure

Figure 2. The schematic diagram of the antenna structure

In order to improve the antenna's working bandwidth, we use the microcosmic fractal structure in the dipole antenna arm; replace dipole antenna arm's small squares area which the size is $1.5 \mathrm{~mm} \times 1.5 \mathrm{~mm}$ with 3 rd-order Hilbert fractal structure which line width is $0.1 \mathrm{~mm}$. In the macroscopic view, the antenna's radiation patch remained the shape of folded dipole antenna; in the microscopic view, each $1.5 \mathrm{~mm} \times 1.5 \mathrm{~mm}$ small squares metal area is a micro 3rd-order Hilbert fractal structure. The metal radiation area inside have self-similar structure, the antenna's working bandwidth will extend greatly.

A novel PBG structure is introduced on the grounded plane to improve the performance of antenna. This PBG structure can be regarded as metal square slices periodic distribution in ceramic substrate. The PBG structure is constituted by four big metal square slices and five small metal square slices, these metal square slices will 
absorb the radiation energy generated by the dipole antenna arms, and produce new radiation signals by induction radiation. We use the radiation of the PBG structure to cover the $1.710-1.785 \mathrm{GHz}, 1.805-1.880 \mathrm{GHz}, 1.880-1.920 \mathrm{GHz}, 1.920-1.980 \mathrm{GHz}$, 2.010-2.025 GHz, 2.110-2.170 GHz, 2.300-2.390 GHz and 2.555-2.655 GHz frequency bands. The frequency of induction radiation signals $f_{r}$ and the edge length of metal square slice $L$ should satisfy the formula (2).

$$
L=\frac{c}{4 f_{r} \sqrt{\varepsilon_{r}}}-0.412 h \frac{\left(\varepsilon_{r}+0.3\right)\left[\frac{c}{2 f_{r} h}\left(\frac{\varepsilon_{r}+1}{2}\right)^{-1 / 2}+0.264\right]}{\left(\varepsilon_{r}-0.258\right)\left[\frac{c}{2 f_{r} h}\left(\frac{\varepsilon_{r}+1}{2}\right)^{-1 / 2}+0.8\right]}
$$

Assigning the light velocity $c=3.0 \times 10^{8} \mathrm{~m} / \mathrm{s}$, the $\varepsilon_{r}=20$ and $h=4 \mathrm{~mm}$, when $f_{r}=$ $1.80 \mathrm{GHz}$, the $L=7.75 \mathrm{~mm}$; when $f_{r}=2.60 \mathrm{GHz}$, the $L=4.72 \mathrm{~mm}$. So we use big and small metal square slices which sizes are $8 \mathrm{~mm} \times 8 \mathrm{~mm}$ and $4 \mathrm{~mm} \times 4 \mathrm{~mm}$ respectively.

\section{The Simulation Analysis of the Performance of Antenna}

By finite-difference time-domain method (FDTDM), the simulated return loss and radiation pattern of designed antenna, with $\varepsilon_{r}=20$ and $h=4 \mathrm{~mm}$, are given in Figure 3 and Figure 4 respectively.

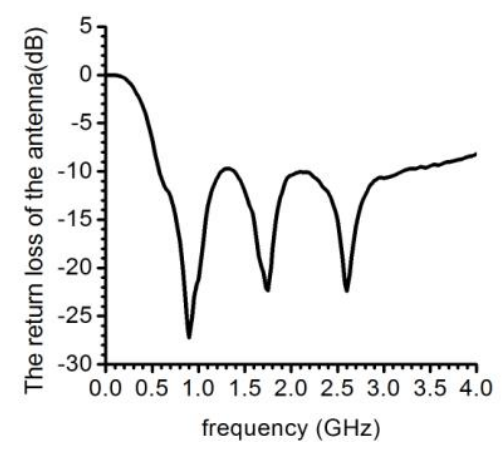

Figure 3. The simulated return loss value of antenna 


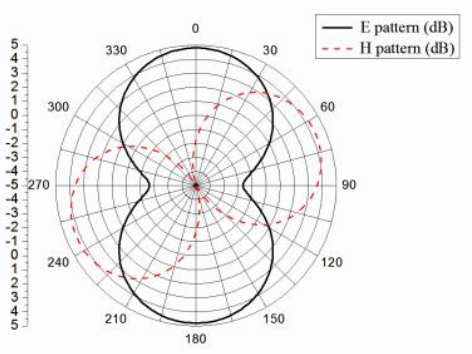

(a) Lower frequency band $(0.90 \mathrm{GHz})$

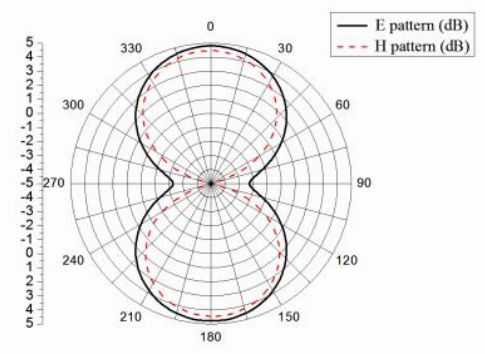

(b) Middle frequency band $(1.75 \mathrm{GHz})$

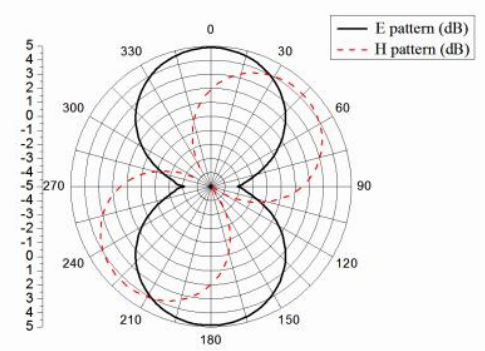

(c) Higher frequency band $(2.60 \mathrm{GHz})$

Figure 4. The simulated radiation pattern of antenna

Figure 3 shows that there are three working center frequency, $0.90 \mathrm{GHz}, 1.75 \mathrm{GHz}$ and $2.60 \mathrm{GHz}$ respectively, at which the return loss values $S_{11}$ are $-27.22 \mathrm{~dB},-22.31 \mathrm{~dB}$ and $-22.36 \mathrm{~dB}$ respectively. For VSWR less than 2 , the absolute working bandwidths are up to $0.667 \mathrm{GHz}(0.584 \sim 1.251 \mathrm{GHz}), 0.688 \mathrm{GHz}(1.398 \sim 2.086 \mathrm{GHz})$ and 1.112 $\mathrm{GHz}(2.086 \sim 3.198 \mathrm{GHz})$, also the relatively working bandwidth up to $72.69 \%, 39.49 \%$ and $42.09 \%$ respectively. All the results show that the return loss characteristic and the working bandwidth can satisfy the request of $2 \mathrm{G}, 3 \mathrm{G}$ and $4 \mathrm{G}$ systems.

According to E-plane radiation pattern and $\mathrm{H}$-plane radiation pattern at $0.90 \mathrm{GHz}$, $1.75 \mathrm{GHz}$ and $2.60 \mathrm{GHz}$, it is obvious that this antenna has hemisphere directional radiation characteristic. The radiation pattern at different frequency have different rotation angle, it is due to the radiation signal in lower frequency band is generated by the dipole antenna arms, but the radiation signal in middle and higher frequency bands are generated by the big and small metal square slices of PBG structure.

In order to make better use of this antenna in real process, the influences of the square PBG slice size on the performance of antenna have been discussed in detail. While changing the square PBG slice size, we keep $h=4 \mathrm{~mm}$ and $\varepsilon_{r}=20$, made a series of simulation calculating, and the result is listed in Table 1.

The simulated results show that appropriate square PBG slice size would be benefit for the working bandwidth and radiation characteristic of antenna. When the size of small square slice close to the dipole antenna arm's width, like $3 \mathrm{~mm} \times 3 \mathrm{~mm}$, the gain of antenna is to be enhanced, it especially for lower frequency band. 
Table 1. Influence of the PBG slice size on antenna performance

\begin{tabular}{|c|c|c|c|c|c|c|c|c|c|c|}
\hline $\begin{array}{c}\text { big } \\
\text { square } \\
\text { slice } \\
\text { size }(\mathrm{mm})\end{array}$ & $\begin{array}{c}\text { small } \\
\text { square } \\
\text { slice } \\
\text { size }(\mathrm{mm})\end{array}$ & $\begin{array}{c}\text { working } \\
\text { center } \\
\text { frequency } \\
1(\mathrm{GHz})\end{array}$ & $\begin{array}{c}S_{11} \\
(\mathrm{~dB})\end{array}$ & $\begin{array}{c}\text { bandwidth } \\
\text { (GHz) }\end{array}$ & $\begin{array}{c}\text { working } \\
\text { center } \\
\text { frequency } \\
2(\mathrm{GHz})\end{array}$ & $\begin{array}{c}S_{11} \\
\text { (dB) }\end{array}$ & $\begin{array}{c}\text { bandwidth } \\
\text { (GHz) }\end{array}$ & $\begin{array}{c}\text { working } \\
\text { center } \\
\text { frequency } \\
3(\mathrm{GHz})\end{array}$ & $\begin{array}{c}S_{11} \\
(\mathrm{~dB})\end{array}$ & $\begin{array}{c}\text { bandwidth } \\
\text { (GHz) }\end{array}$ \\
\hline $7 \times 7$ & $3 \times 3$ & 0.90 & -37.44 & 0.776 & 1.75 & -21.20 & 0.867 & 2.60 & -21.35 & 0.843 \\
\hline $7 \times 7$ & $4 \times 4$ & 0.90 & -35.02 & 0.750 & 1.75 & -22.68 & 0.862 & 2.60 & -21.45 & 0.734 \\
\hline $7 \times 7$ & $5 \times 5$ & 0.90 & -28.38 & 0.678 & 1.75 & -22.96 & 0.735 & 2.60 & -21.64 & 0.626 \\
\hline $8 \times 8$ & $3 \times 3$ & 0.90 & -31.06 & 0.771 & 1.75 & -20.74 & 0.836 & 2.60 & -22.07 & 1.229 \\
\hline $8 \times 8$ & $4 \times 4$ & 0.90 & -27.22 & 0.667 & 1.75 & -22.31 & 0.688 & 2.60 & -22.36 & 1.112 \\
\hline$\underline{8 \times 8}$ & $\underline{5 \times 5}$ & $\underline{0.90}$ & -27.20 & $\underline{0.663}$ & $\underline{1.75}$ & -22.47 & $\underline{0.685}$ & $\underline{2.60}$ & -22.47 & $\underline{1.092}$ \\
\hline $9 \times 9$ & $3 \times 3$ & 0.90 & -30.26 & 0.742 & 1.75 & -20.63 & 0.801 & 2.60 & -23.01 & 0.747 \\
\hline $9 \times 9$ & $4 \times 4$ & 0.90 & -26.91 & 0.657 & 1.75 & -21.59 & 0.687 & 2.60 & -23.05 & 0.570 \\
\hline $9 \times 9$ & $\underline{5 \times 5}$ & $\underline{0.90}$ & -25.94 & 0.653 & $\underline{1.75}$ & -22.11 & 0.616 & 2.60 & -23.21 & 0.564 \\
\hline
\end{tabular}

\section{The Fabrication and Test of Antenna Sample}

According to the design mentioned above, we fabricate the tri-frequency microcosmic fractal dipole antenna sample, which is shown in Figure 5.

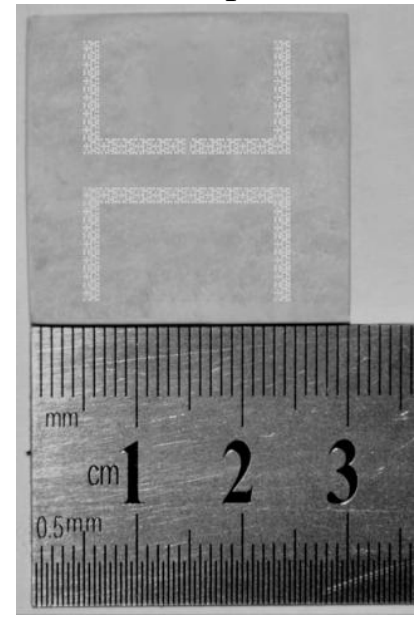

(a) Microcosmic fractal dipole antenna structure

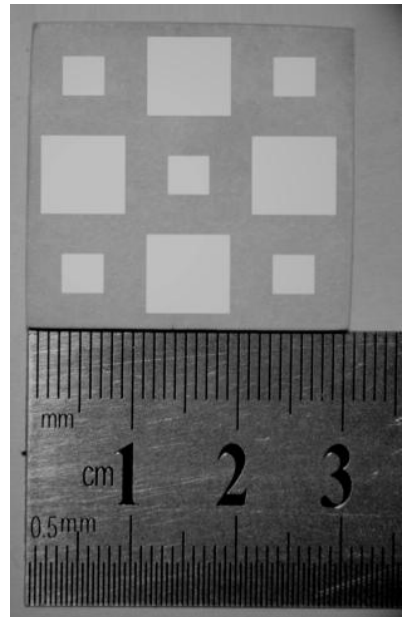

(b) PBG structure

Figure 5. The photo of antenna sample

We use RF integrated vector network analyzer to test the return loss of the antenna, with the result is shown in Figure 6; we set up an open area test site (OATS) to test antenna's radiation pattern characteristics, with the result is shown in Figure 7.

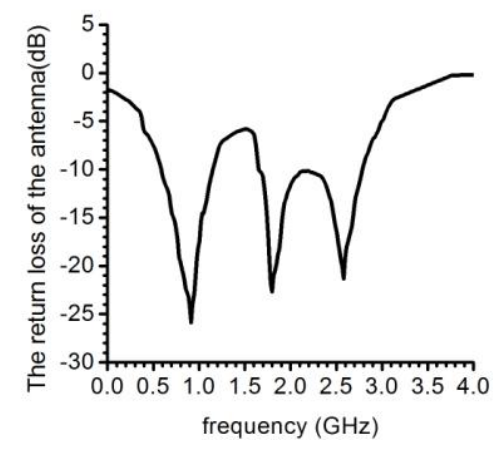

Figure 6 . The tested return loss value of antenna 


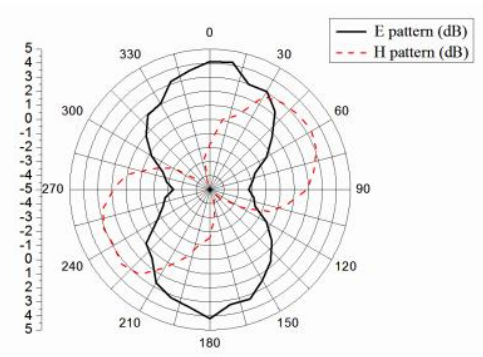

(a) Lower frequency band $(0.92 \mathrm{GHz})$

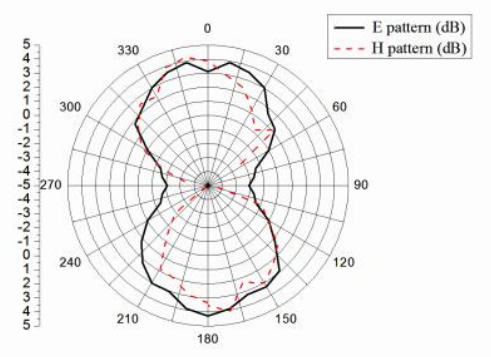

(b) Middle frequency band $(1.80 \mathrm{GHz})$

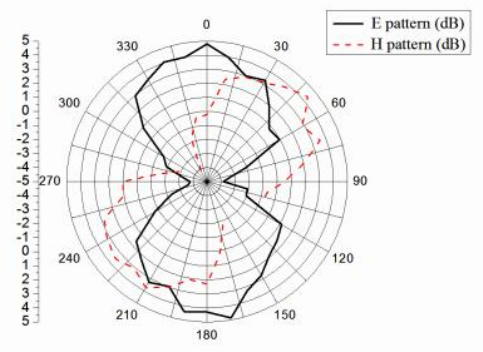

(c) Higher frequency band $(2.58 \mathrm{GHz})$

Figure 7 . The tested radiation pattern of antenna

The tested results show that there are three working center frequency, $0.92 \mathrm{GHz}, 1.80$ $\mathrm{GHz}$ and $2.58 \mathrm{GHz}$ respectively, at which the return loss values $S_{11}$ are $-25.85 \mathrm{~dB}$, $-22.66 \mathrm{~dB}$ and $-21.32 \mathrm{~dB}$ respectively. For VSWR less than 2, the absolute working bandwidths are up to $0.567 \mathrm{GHz}(0.586 \sim 1.153 \mathrm{GHz}), 0.544 \mathrm{GHz}(1.648 \sim 2.192 \mathrm{GHz})$ and $0.592 \mathrm{GHz}(2.192 \sim 2.784 \mathrm{GHz})$, also the relatively working bandwidth up to $65.21 \%, 28.33 \%$ and $23.79 \%$ respectively. According to E-plane radiation pattern and H-plane radiation pattern at $0.92 \mathrm{GHz}, 1.80 \mathrm{GHz}$ and $2.58 \mathrm{GHz}$, this antenna has hemisphere directional radiation characteristic.

In a whole, the tested and simulated results are similar, but the tested radiation characteristic, bandwidth performance and radiation patterns with a little worse. It is due to that the manufacturing error and welding in engineering processes cause the antenna to have lost matching a little.

\section{The Design of Improved Antenna Structure}

To improve the antenna performance in further, we tried an effective ways and did some comparative analysis. This way is to apply fractal structure in the designed antenna's PBG structure. Here, each square slice of PBG structure is replaced by a 2nd-order Cantor fractal structure, the scheme of which is shown in Figure 8. 


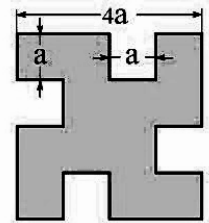

(a) 1st-order

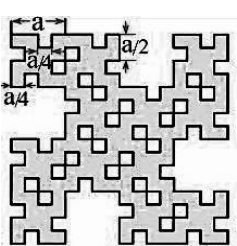

(b) 2nd-order

Figure 8. The iteration process of Cantor fractal structure

By simulation, two curves in Figure 9 present the return loss of antenna with 2nd-order Cantor fractal structure and without fractal structure in square PBG slices respectively.

It can be seen that the application of fractal structure can improve the radiation characteristics of antenna and broaden the working bandwidth obviously. Applying this improved structure, the antenna have three working center frequency, $0.90 \mathrm{GHz}, 1.75$ $\mathrm{GHz}$ and $2.64 \mathrm{GHz}$ respectively, at which the return loss values $S_{11}$ are $-25.04 \mathrm{~dB}$, $-20.83 \mathrm{~dB}$ and $-24.84 \mathrm{~dB}$ respectively. For VSWR less than 2, the absolute working bandwidths are up to $0.946 \mathrm{GHz}(0.506 \sim 1.452 \mathrm{GHz}), 0.722 \mathrm{GHz}(1.452 \sim 2.174 \mathrm{GHz})$ and $1.164 \mathrm{GHz}(2.174 \sim 3.338 \mathrm{GHz})$, also the relatively working bandwidth up to $96.63 \%, 39.82 \%$ and $42.24 \%$ respectively.

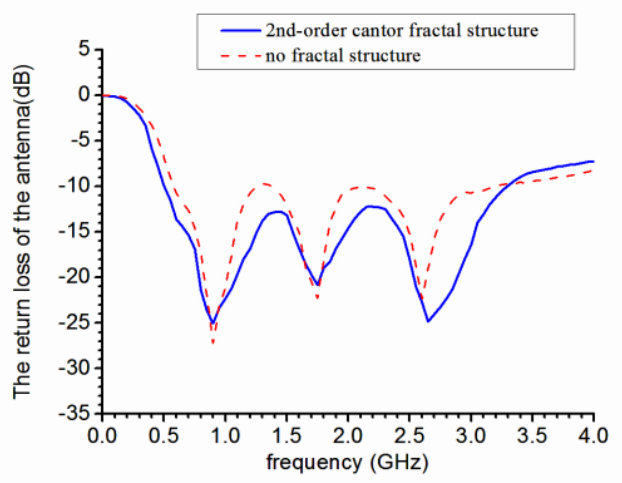

Figure 9. The return loss values of designed antenna with 2nd-order Cantor fractal structure and without fractal structure

\section{Conclusions}

First of all, the structure design of a tri-frequency microcosmic fractal dipole antenna with PBG structure is introduced in this paper. Simulated and tested results show that this antenna has a small size, large bandwidth, good return loss characteristic and hemisphere direction radiation characteristic. This antenna can completely covered the 0.905-0.915 GHz, 0.950-0.960 GHz, 1.710-1.785 GHz, 1.805-1.880 GHz, 1.880-1.920 $\mathrm{GHz}, 1.920-1.980 \mathrm{GHz}, 2.010-2.025 \mathrm{GHz}, 2.110-2.170 \mathrm{GHz}, 2.300-2.390 \mathrm{GHz}$ and 2.555-2.655 GHz communication frequency bands, it successfully achieved the compatibility of the GSM, TD-SCDMA, WCDMA, TD-LTE mobile communications standard. More discussion suggests that appropriate square PBG slice size would be benefit for the bandwidth and radiation characteristic of antenna. At last, the application of Cantor fractal structure in PBG structure has been tried for the designed antenna successfully. This antenna can be applied in the 2G/3G/4G mobile communication systems, and thus has broad application prospects. 


\section{Acknowledgement}

The project was supported by the Natural Science Foundation of Fujian Province of China (No. 2016J01318).

\section{References}

[1] A Amsavalli, KR Kashwan. Smart Patch Antenna Array for Uplink in 4G Mobile Communication Based on LMS Algorithm for DS-CDMA Technique [J]. Journal of Convergence Information Technology, 2014, 9(1): 16-24.

[2] Younes Karfa Bekali, Mohamed Essaaidi. Compact reconfigurable dual frequency microstrip patch antenna for $3 \mathrm{G}$ and $4 \mathrm{G}$ mobile communication technologies [J]. Microwave and Optical Technology Letters, 2013, 55(7): 1622-1626.

[3] Elvis Kobina Donkoh, Samuel K Amponsah, Alex A. Opoku, et al. Hexagonal Tessellation Model for Masting GSM Antenna: Case Study of MTN Kumasi East-Ghana [J]. IAENG International Journal of Applied Mathematics, 2015, 30(1): 1349-1357. 
[4] Yujie Liu, Wei Tang, Yuehe Ge, et al. A Compact Wideband Dual-Polarized Printed Antenna with Coaxial Feeds for TD-SCDMA Application [J]. Wireless Engineering and Technology, 2015, 6(1): 1-7.

[5] Shaojian Chen, Dacheng Dong, Zhouying Liao, et al. Compact wideband and dual-band antenna for TD-LTE and WLAN applicationss [J]. Electronics Letters, 2014, 50(16): 1111-1112.

[6] Omid Kaboli, Ali Gashtasbi, Alireza Monajati. Design ,Simulation ,Fabrication and Measurement of 900MHZ Newhybrid Fractal Dipole Antenna [J]. International Journal of Electronics Communication and Computer Engineering, 2015, 6(1): 20-22.

[7] Zhinong Ying. Antennas in Cellular Phones for Mobile Communications [J]. Proceedings of the IEEE, 2012, 100(7): 2286-2296.

[8] Junho Yeo, Jong-Ig Lee. Broadband series-fed two dipole array antenna with an integrated balun for mobile communication applications [J]. Microwave and Optical Technology Letters, 2012, 54(9): 2166-2168.

[9] Yu-Fa Zheng, Sai-Wai Wong, Qi-Kai Huang, et al. Wideband dipole antenna using multiple-mode resonator for long-term evolution application $[\mathrm{J}]$. Electronics Letters, 2015, 51(25): 2074-2076.

[10] Yejun He, Wei He, Hang Wong. A Wideband Circularly Polarized Cross-Dipole Antenna [J]. IEEE Antennas and Wireless Propagation Letters, 2014, 13(1): 67-70.

[11] Mingjian Li, Kwai-Man Luk. A Differential-Fed Magneto-Electric Dipole Antenna for UWB Applications [J]. IEEE Transactions on Antennas and Propagation, 2013, 61(1): 92-99.

[12] Jonathan P. P. Pereira, José P. da Silva, Humberto D. de Andrade. A new design and analysis of a hexagonal PBG microstrip antenna [J]. Microwave and Optical Technology Letters, 2015, 57(9): 2147-2151.

[13] Hong Yang, Zhipeng Wang, Jianxing Shao. Analysis and Design of a Multi-Frequency Microstrip Antenna Based on a PBG Substrate [J]. Sensors \& Transducers, 2014, 172(6): 178-183.

[14] Yadwinder Kumar, Surinder Singh. Microstrip Fed Multiband Hybrid Fractal Antenna for Wireless Applications [J]. Applied Computational Electromagnetics Society Journal, 2016, 31(3): 327-332.

[15] Shweta Rani, A.P Singh. A novel design of hybrid fractal antenna using BFO [J]. Journal of Intelligent \& Fuzzy Systems, 2014, 27(3): 1233-1241.

[16] Nitin Saluja, Rajesh Khanna. Design analysis and fabrication of novel coplanar waveguide-fed hybrid fractal-based Broadband antenna [J]. International Journal of Microwave and Wireless Technologies, 2013, 5(6): 749-752. 